\title{
QUANTUM FOAM
}

\author{
Y. JACK NG \\ Institute of Field Physics, Department of Physics and Astronomy, \\ University of North Carolina, Chapel Hill, NC 27599-3255, USA \\ E-mail: yjng@physics.unc.edu
}

\begin{abstract}
Quantum foam, also known as spacetime foam, has its origin in quantum fluctuations of spacetime. Its physics is intimately linked to that of black holes and computation. Arguably it is the source of the holographic principle which severely limits how densely information can be packed in space. Various proposals to detect the foam are briefly discussed. Its detection will provide us with a glimpse of the ultimate structure of space and time.
\end{abstract}

\section{Introduction}

Before last century, spacetime was regarded as nothing more than a passive and static arena in which events took place. Early last century, Einstein's general relativity changed that viewpoint and promoted spacetime to an active and dynamical entity. Nowadays many physicists also believe that spacetime, like all matter and energy, undergoes quantum fluctuations. These quantum fluctuations make spacetime foamy ${ }^{\mathrm{a}}$ on small spacetime scales.

But how large are the fluctuations? How foamy is spacetime? Is there any theoretical evidence of quantum foam? And how can we detect quantum foam? In what follows, we address these questions. The outline of this paper is as follows: By analysing a gedanken experiment for spacetime measurement, we show, in subsection 2.1, that spacetime fluctuations scale as the cube root of distances or time durations. In subsection 2.2, we show that this cube root dependence is consistent with the holographic principle. Subsection 2.3 is devoted to a comparison of this peculiar dependence with the well-known random-walk problem and other quantum gravity models. Here we also consider the cumulative effects of individual spacetime fluctuations. In section 3 , we discuss how quantum foam affects the physics of clocks and computation (subsection 3.1), and show that the physics of spacetime foam is intimately connected to that of black holes (subsection 3.2). Just as there are uncertainties in spacetime measurements, there are also uncertainties in energy-momentum measurements. This topic of energy-momentum uncertainties is given a brief treatment in section 4 . Some proposals to detect quantum foam are

$\overline{{ }^{\mathrm{a}} \text { For a brief review and a more complete list of references, see Ref. } 1 .}$ 
considered in section 5. One particular proposal involving ultra-high energy cosmic ray events is discussed in the Appendix.

Before we proceed, we should mention that the approach to the physics of quantum foam adopted here is very conservative: the only ingredients we use are quantum mechanics and general relativity. Hopefully, by considering only distances (time durations) much larger than the Planck length (time) or energies (momenta) much smaller than Planck energy (momentum), a semi-classical treatment of gravity suffices and a bona fide theory of quantum gravity is not needed. We should also make it clear at the outset that we make no assumptions on the high energy regime of the ultimate quantum gravity theory. We refrain from speculating on violations of Lorentz invariance and the consequent systematically modified dispersion relations, involving a coefficient of fixed magnitude and fixed sign, which many people believe are unavoidably induced by quantum gravity. (In the terminology of Ref. 2, these quantum gravity effects are called "systematic" effects.) The only quantum gravity effects we are concerned with in this paper are those due to quantum fuzziness - uncertainties involving fluctuating magnitudes with both \pm signs, perhaps like a fluctuation with a Gaussian distribution about zero. (In the terminology of Ref. 2, these effects are called "non-systematic" effects.)

\section{Quantum Fluctuations of Spacetime}

If spacetime indeed undergoes quantum fluctuations, the fluctuations will show up when we measure a distance (or a time duration), in the form of uncertainties in the measurement. Conversely, if in any distance (or time duration) measurement, we cannot measure the distance (or time duration) precisely, we interpret this intrinsic limitation to spacetime measurements as resulting from fluctuations of spacetime.

The question is: does spacetime undergo quantum fluctuations? And if so, how large are the fluctuations? To quantify the problem, let us consider measuring a distance $l$. The question now is: how accurately can we measure this distance? Let us denote by $\delta l$ the accuracy with which we can measure $l$. We will also refer to $\delta l$ as the uncertainty or fluctuation of the distance $l$ for reasons that will become obvious shortly. We will show that $\delta l$ has a lower bound and will use two ways to calculate it. Neither method is rigorous, but the fact that the two very different methods yield the same result bodes well for the robustness of the conclusion.

\subsection{Gedanken Experiment}

In the first method, we conduct a thought experiment to measure $l$. The importance of carrying out spacetime measurements to find the quantum fluctuations in the fabric of spacetime cannot be over-emphasized. According to general relativity, coordinates do not have any intrinsic meaning independent of observations; a coordinate system is defined only by explicitly carrying out spacetime distance measurements. Let us measure the distance between point A and point B. Following 
Wigner ${ }^{3}$, we put a clock at A and a mirror at B. Then the distance $l$ that we want to measure is given by the distance between the clock and the mirror. By sending a light signal from the clock to the mirror in a timing experiment, we can determine the distance $l$. However, the quantum uncertainty in the positions of the clock and the mirror introduces an inaccuracy $\delta l$ in the distance measurement. We expect the clock and the mirror to contribute comparable uncertainties to the measurement. Let us concentrate on the clock and denote its mass by $m$. Wigner argued that if it has a linear spread $\delta l$ when the light signal leaves the clock, then its position spread grows to $\delta l+\hbar l(m c \delta l)^{-1}$ when the light signal returns to the clock, with the minimum at $\delta l=(\hbar l / m c)^{1 / 2}$. Hence one concludes that

$$
\delta l^{2} \gtrsim \frac{\hbar l}{m c}
$$

Thus quantum mechanics alone would suggest using a massive clock to reduce the jittering of the clock and thereby the uncertainty $\delta l$. On the other hand, according to general relativity, a massive clock would distort the surrounding space severely, affecting adversely the accuracy in the measurement of the distance.

It is here that we appreciate the importance of taking into account the effects of instruments in this thought-experiment. Usually when one wants to examine a certain a field (say, an electromagnetic field) one uses instruments that are neutral (electromagnetically neutral) and massive for, in that case, the effects of the instruments are negligible. But here in our thought-experiment, the relevant field is the gravitational field. One cannot have a gravitationally neutral yet massive set of instruments because the gravitational charge is equal to the mass according to the principle of equivalence in general relativity. Luckily for us, we can now exploit this equality of the gravitational charge and the inertial mass of the clock to eliminate the dependence on $m$ in the above inequality to promote Eq. (11) to a (low-energy) quantum gravitational uncertainty relation.

To see this, let the clock be a light-clock consisting of a spherical cavity of diameter $d$, surrounded by a mirror wall of mass $m$, between which bounces a beam of light. For the uncertainty in distance measurement not to be greater than $\delta l$, the clock must tick off time fast enough that $d / c \lesssim \delta l / c$. But $d$, the size of the clock, must be larger than the Schwarzschild radius $r_{S} \equiv 2 G m / c^{2}$ of the mirror, for otherwise one cannot read the time registered on the clock. From these two requirements, it follows that

$$
\delta l \gtrsim \frac{G m}{c^{2}}
$$

Thus general relativity alone would suggest using a light clock to do the measurement. This result can also be derived in another way. If the clock has a radius $d / 2$ (larger than its Schwarzschild radius $r_{S}$ ), then $\delta l$, the error in the distance measurement caused by the curvature generated by the mass of the clock, may be estimated by a calculation from the Schwarzschild solution. The result is $r_{S}$ mul- 
tiplied by a logarithm involving $2 r_{S} / d$ and $r_{S} /(l+d / 2)$. For $d>>r_{S}$, one finds $\delta l=\frac{1}{2} r_{S} \log \frac{d+2 l}{d}$ and hence Eq. (2) as an order of magnitude estimate.

The product of Eq. (2) with Eq. (11) yields

$$
\delta l \gtrsim\left(l l_{P}^{2}\right)^{1 / 3}=l_{P}\left(\frac{l}{l_{P}}\right)^{1 / 3},
$$

where $l_{P}=\left(\hbar G / c^{3}\right)^{1 / 2}$ is the Planck length. (Note that the result is independent of the mass of the clock and, thereby, one would hope, of the properties of the specific clock used in the measurement.) The end result is as simple as it is strange and appears to be universal: the uncertainty $\delta l$ in the measurement of the distance $l$ cannot be smaller than the cube root of $l l_{P}^{2} \stackrel{4}{4}$ Obviously the accuracy of the distance measurement is intrinsically limited by this amount of uncertainty or quantum fluctuation. We conclude that there is a limit to the accuracy with which one can measure a distance; in other words, we can never know the distance $l$ to a better accuracy than the cube root of $l l_{P}^{2}$. (Similarly one can show that we can never know a time duration $\tau$ to a better accuracy than the cube root of $\tau t_{P}^{2}$, where $t_{P} \equiv l_{P} / c$ is the Planck time. $)^{\mathrm{b}}$ Because the Planck length is so inconceivably short, the uncertainty or intrinsic limitation to the accuracy in the measurement of any distance, though much larger than the Planck length, is still very small. For example, in the measurement of a distance of one kilometer, the uncertainty in the distance is to an atom as an atom is to a human being.

\subsection{The Holographic Principle}

Alternatively we can estimate $\delta l$ by applying the holographic principle $\frac{67}{6}$ In essence, the holographic principle ${ }^{8}$ says that although the world around us appears to have three spatial dimensions, its contents can actually be encoded on a twodimensional surface, like a hologram. To be more precise, let us consider a spatial region measuring $l$ by $l$ by $l$. According to the holographic principle, the number of degrees of freedom that this cubic region can contain is bounded by the surface area of the region in Planck units, i.e., $l^{2} / l_{P}^{2}$, instead of by the volume of the region as one may naively expect. This principle is strange and counterintuitive, but is supported by black hole physics in conjunction with the laws of thermodynamics, and it is embraced by both string theory and loop gravity, two top contenders of quantum gravity theory. So strange as it may be, let us now apply the holographic principle to deduce the accuracy with which one can measure a distance.

First, imagine partitioning the big cube into small cubes [see Fig. 囵. The small cubes so constructed should be as small as physical laws allow so that we can associate one degree of freedom with each small cube. In other words, the number

\footnotetext{
${ }^{\mathrm{b}}$ The spacetime fluctuation translates into a metric fluctuation over a distance $l$ and a time interval $\tau$ given by $\delta g_{\mu \nu}$ greater than $\left(l_{P} / l\right)^{2 / 3},\left(t_{P} / \tau\right)^{2 / 3}$ respectively. For a discussion of the related lightcone fluctuations, see Ref. 5 .
} 


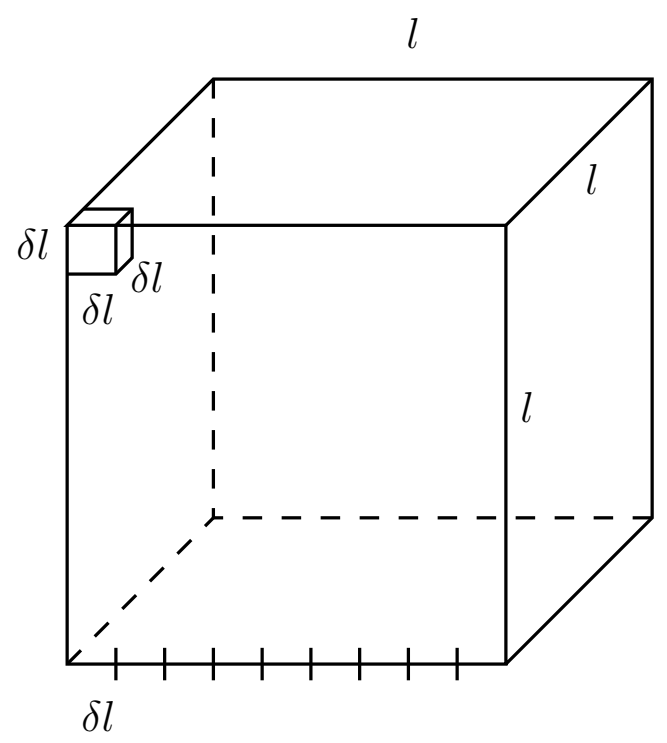

Figure 1. Partitioning a big cube into small cubes. The big cube represents a region of space measuring $l$ by $l$ by $l$. The small cubes represent the smallest physically-allowed cubes measuring $\delta l$ by $\delta l$ by $\delta l$ that can be lined up to measure the length of each side of the big cube. Strangely, the size of a small cube is not universal, but depends on the size of the big cube. A simple argument based on this construction leads to the holographic principle.

of degrees of freedom that the region can hold is given by the number of small cubes that can be put inside that region. But how small can such cubes be? A moment's thought tells us that each side of a small cube cannot be smaller than the accuracy $\delta l$ with which we can measure each side $l$ of the big cube. This can be easily shown by applying the method of contradiction: assume that we can construct small cubes each of which has sides less than $\delta l$. Then by lining up a row of such small cubes along a side of the big cube from end to end, and by counting the number of such small cubes, we would be able to measure that side (of length $l$ ) of the big cube to a better accuracy than $\delta l$. But, by definition, $\delta l$ is the best accuracy with which we can measure $l$. The ensuing contradiction is evaded by the realization that each of the smallest cubes (that can be put inside the big cube) measures $\delta l$ by $\delta l$ by $\delta l$. Thus, the number of degrees of freedom in the region (measuring $l$ by $l$ by $l$ ) is given by $l^{3} / \delta l^{3}$, which, according to the holographic principle, is no more than $l^{2} / l_{p}^{2}$. It follows that $\delta l$ is bounded (from below) by the cube root of $l l_{P}^{2}$, the same result as found above in the gedanken experiment argument. Thus, to the extent that the holographic principle is correct, spacetime indeed fluctuates, forming foams of size $\delta l$ on the scale of $l$. Actually, considering the fundamental nature of spacetime and the ubiquity of quantum fluctuations, we should reverse the argument and then we will come to the conclusion that the "strange" holographic principle has its origin 
in quantum fluctuations of spacetime. ${ }^{c}$

\subsection{Quantum Gravity Models}

The consistency of the uncertainties in distance measurements with the holographic principle is reassuring. But the dependence of the fluctuations in distance on the cube root of the distance is still perplexing. To gain further insight into this strange state of affairs, let us compare this peculiar dependence on distance with the wellknown one-dimensional random-walk problem. For a random walk of steps of equal size, with each step equally likely to either direction, the root-mean-square deviation from the mean is given by the size of each step multiplied by the square root of the number of steps. It is now simple to concoct a random-walk mode 1011 for the fluctuations of distances in quantum gravity. Consider a distance $l$, which we partition into $l / l_{P}$ units each of length $l_{P}$. In the random-walk model of quantum gravity, $l_{P}$ plays the role of the size of each step and $l / l_{P}$ plays the role of the number of steps. The fluctuation in distance $l$ is given by $l_{P}$ times the square root of $l / l_{P}$, which comes out to the square root of $l l_{P}$. This is much bigger than the cube root of $l l_{P}^{2}$, the fluctuation in distance measurements found above.

The following interpretation of the dependence of $\delta l$ on the cube root of $l$ now presents itself. As in the random-walk model, the amount of fluctuations in the distance $l$ can be thought of as an accumulation of the $l / l_{P}$ individual fluctuations each by an amount plus or minus $l_{P}$. But, for this case, the individual fluctuations cannot be completely random (as opposed to the random-walk model); actually successive fluctuations must be somewhat anti-correlated (i.e., a plus fluctuation is slightly more likely followed by a minus fluctuation and vice versa), in order that together they produce a total fluctuation less than that in the random-walk model. This small amount of anti-correlation between successive fluctuations (corresponding to what statisticians call fractional Brownian motion with self-similarity parameter $\frac{1}{3}$ ) must be due to quantum gravity effects. Since the cube root dependence on distance has been shown to be consistent with the holographic principle, we will, for the rest of this subsection, refer to this case that we have found (marked by an arrow in Fig. (2) as the holography model.

On the other hand, if successive fluctuations are completely anti-correlated, i.e., a fluctuation by plus $l_{P}$ is followed by a fluctuation by minus $l_{P}$ which is succeeded by plus $l_{P}$ etc. in the pattern $+-+-+-+-+-\ldots$, then the fluctuation of a distance $l$ is given by the minuscule $l_{P}, \frac{12}{12}$ independent of the size of the distance. Thus the holography model falls between the two extreme cases of complete randomness (square root of $l l_{P}$ ) and complete anti-correlation $\left(l_{P}\right)$. For completeness, we mention that a priori there are also models with correlating successive fluctuations. But these models yield unacceptably large fluctuations in

${ }^{\mathrm{c}}$ Recently, Scardigli and Casadid ${ }^{9}$ claim that the expected holographic scaling seems to hold only in $(3+1)$ dimensions and only for the "generalized uncertainty principle" found above for $\delta l$. 
distance and time duration measurements - we will see below that these models (corresponding to the hatched line to the right of the random-walk model shown in Fig. (2) have already been observationally ruled out.

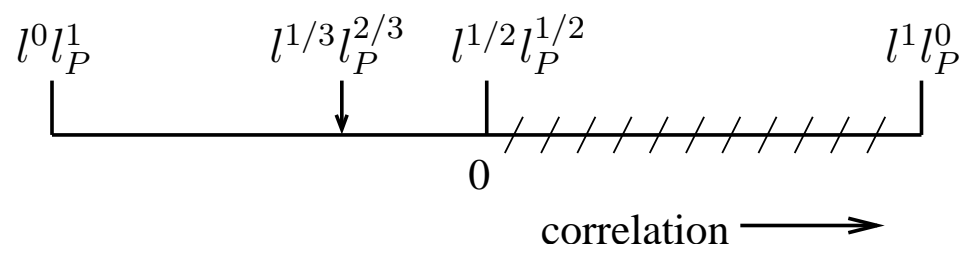

Figure 2. Lower bounds on $\delta l$ for the various quantum gravity models. The fluctuation of the distance $l$ is given by the sum of $l / l_{P}$ fluctuations each by plus or minus $l_{P}$. Spacetime foam appears to choose a small anti-correlation (i.e., negative correlation) between successive fluctuations, giving a cube root dependence in the number $l / l_{p}$ of fluctuations for the total fluctuation of $l$ (indicated by the arrow). It falls between the two extreme cases of complete randomness, i.e., zero (anti-)correlation (corresponding to $\delta l \sim l^{1 / 2} l_{P}^{1 / 2}$ ) and complete anti-correlation (corresponding to $\delta l \sim l_{P}$ ). Quantum gravity models corresponding to positive correlations between successive fluctuations (indicated by the hatched portion) are observationally ruled out.

Let us now examine the cumulative effects 13 of spacetime fluctuations over a large distance. Consider a distance $l$, and divide it into $l / \lambda$ equal parts each of which has length $\lambda$. If we start with $\delta \lambda$ from each part, the question is how do the $l / \lambda$ parts add up to $\delta l$ for the whole distance $l$. In other words, we want to find the cumulative factor $\mathcal{C}$ defined by

$$
\delta l=\mathcal{C} \delta \lambda,
$$

For the holography model, since $\delta l \sim l^{1 / 3} l_{P}^{2 / 3}=l_{P}\left(l / l_{P}\right)^{1 / 3}$ and $\delta \lambda \sim \lambda^{1 / 3} l_{P}^{2 / 3}=$ $l_{P}\left(\lambda / l_{P}\right)^{1 / 3}$, the result is

$$
\mathcal{C}=\left(\frac{l}{\lambda}\right)^{1 / 3}
$$

For the random-walk model, the cumulative factor is given by $\mathcal{C}=(l / \lambda)^{1 / 2}$; for the model corresponding to complete anti-correlation, the cumulative factor is $\mathcal{C}=1$, independent of $l$. Let us note that, for all quantum gravity models (except for the physically disallowed model corresponding to complete correlation between successive fluctuations), the cumulative factor is not linear in $(l / \lambda)$, i.e., $\frac{\delta l}{\delta \lambda} \neq \frac{l}{\lambda}$. (In fact, it is much smaller than $l / \lambda$ ). The reason for this is obvious: the $\delta \lambda$ 's from the $l / \lambda$ parts in $l$ do not add coherently. It makes no sense, e.g., to say, for the completely anti-correlating model, that $\delta l \sim \delta \lambda \times l / \lambda \gtrsim l_{P} l / \lambda$ because it is inconsistent to use the completely anti-correlating model for $\delta \lambda$ while using the completely correlating model for the cumulative factor.

Note that the above discussion on cumulative effects is valid for any $\lambda$ between $l$ and $l_{P}$, i.e., it does not matter how one partitions the distance $l$. In particular, for our holography model, one can choose to partition $l$ into units of Planck length 
Table 1. The corresponding quantities in the discussion of distance measurements (first column), time duration measurements (second column), clocks (third column), and computers (fourth column) appear in the same row in the following Table.

\begin{tabular}{llll}
\hline $\begin{array}{l}\text { distance } \\
\text { measurements }\end{array}$ & $\begin{array}{l}\text { time duration } \\
\text { measurements }\end{array}$ & clocks & computers \\
\hline \hline distance uncertainty & time duration & resolution & reciprocal of \\
divided by speed & uncertainty & time & computation \\
of light $(\delta l / c)$ & $(\delta \tau)$ & $(t)$ & speed $(1 / \nu)$ \\
\hline distance & time duration & running & number of bits \\
divided by speed & $(\tau)$ & time & divided by compu- \\
of light $(l / c)$ & & $(T)$ & tation speed $(I / \nu)$ \\
\hline
\end{tabular}

$l_{P}$, the smallest physically meaningful length. Then (for $\lambda=l_{P}$ ) using $\delta l_{P} \sim$ $l_{P}^{1 / 3} \times l_{P}^{2 / 3}=l_{P}$, one recovers $\delta l \sim\left(l / l_{P}\right)^{1 / 3} \times l_{P}=l^{1 / 3} l_{P}^{2 / 3}$, with the dependence on the cube root of $l$ being due to a small amount of anti-correlation between successive fluctuations as noted above. The fact that we can choose $\lambda$ as small as the Planck length in the partition indicates that, in spite of our earlier disclaimer, it may even be meaningful to consider, in the semi-classical framework we are pursuing, fluctuations of distances close to the Planck length.

Now that we know where the holography model stands among the quantum gravity models, we will restrict ourselves to discuss this model only for the rest of the paper.

\section{From Spacetime Foam to Computers to Black Holes}

So far there is no experimental evidence for spacetime foam, and, as we will show shortly, no direct evidence is expected in the very near future. In view of this lack of experimental evidence, we should at least look for theoretical corroborations (aside from the "derivation" of the holographic principle discussed above). Fortunately such corroborations do exist - in the sector of black hole physics (this should not come as a surprise to the experts). To show that, we have to make a small detour to consider clocks and computers $\underline{1415}$ first.

\subsection{Clocks and Computers}

Consider a clock (technically, a simple and "elementary" clock, not composed of smaller clocks that can be used to read time separately or sequentially), capable of resolving time to an accuracy of $t$, for a period of $T$ (the running time or lifetime of the clock). Then bounds on the resolution time and the lifetime of the clock can be derived by following an argument very similar to that used above in the analysis of the gedanken experiment to measure distances. Actually, the two arguments are so similar that one can identify the corresponding quantities. [See Table.]

For the discussion of clocks, one argues that at the end of the running time 
$T$, the linear spread of the clock (of mass $m$ ) grows to $\delta l \gtrsim(\hbar T / m)^{1 / 2}$. But the position uncertainty due to the act of time measurement must be smaller than the minimum wavelength of the quanta used to read the clock: $\delta l \lesssim c t$, for the entire period $T$. It follows that 14

$$
t^{2} \gtrsim \frac{\hbar T}{m c^{2}}
$$

which is the analogue of Eq. (11). On the other hand, for the clock to be able to resolve time interval as small as $t$, the cavity of the light-clock must be small enough such that $d \lesssim c t$; but the clock must also be larger than the Schwarzschild radius $2 G m / c^{2}$ so that the time registered by the clock can be read off at all. These two requirements are satisfied with

$$
t \gtrsim \frac{G m}{c^{3}}
$$

the analogue of Eq. (2). One can combine the above two equations to give 14

$$
T / t^{3} \lesssim t_{P}^{-2}=\frac{c^{5}}{\hbar G}
$$

which relates clock precision to its lifetime. Numerically, for example, for a femtosecond $\left(10^{-15} \mathrm{sec}\right)$ precision, the bound on the lifetime of a simple clock is $10^{34}$ years.

One can easily translate the above relations for clocks into useful relations for a simple computer (technically, it refers to a computer designed to perform highly serial computations, i.e., one that is not divided into subsystems computing in parallel). Since the resolution time $t$ for clocks is the smallest time interval relevant in the problem, the fastest possible processing frequency is given by its reciprocal, i.e., $1 / t$. Thus if $\nu$ denotes the clock rate of the computer, i.e., the number of operations per bit per unit time, then it is natural to identify $\nu$ with $1 / t$. To identify the number $I$ of bits of information in the memory space of a simple computer, we recall that the running time $T$ is the longest time interval relevant in the problem. Thus, the maximum number of steps of information processing is given by the running time divided by the resolution time, i.e., $T / t$. It follows that one can identify the number $I$ of bits of the computer with $T / t{ }^{\text {d }}$ In other words, the translations from the case of clocks to the case of computers consist of substituting the clock rate of computation for the reciprocal of the resolution time, and substituting the number of bits for the running time divided by the resolution time. [See Table.] The bound on the precision and lifetime of a clock given by Eq. (8) is now translated into a bound on the rate of computation and number of bits in the computer, yielding

$$
I \nu^{2} \lesssim \frac{c^{5}}{\hbar G} \sim 10^{86} / \sec ^{2}
$$

$\overline{\mathrm{d} O n e}$ can think of a tape of length $c T$ as the memory space, partitioned into bits each of length ct. 
The latter bound is intriguing: it requires the product of the number of bits and the square of the computation rate for any simple computer to be less than the square of the reciprocal of Planck time 14 which depends on relativistic quantum gravity (involving $c, \hbar$, and $G$ ). This relation links together our concepts of information/computation, relativity, gravity, and quantum uncertainty. Numerically, the computation bound is about seventy-six orders of magnitude above what is available for a current lap-top computer performing ten billion operations per second on ten billion bits, for which $I \nu^{2} \sim 10^{10} / \mathrm{s}^{2}$.

\subsection{Black Holes}

Now we can apply what we have learned about clocks and computers to black holes.1415 Let us consider using a black hole to measure time. It is reasonable to use the light travel time around the black hole's horizon as the resolution time of the clock, i.e., $t \sim \frac{G m}{c^{3}} \equiv t_{B H}$, then from Eq. (6), one immediately finds that

$$
T \sim \frac{G^{2} m^{3}}{\hbar c^{4}} \equiv T_{B H}
$$

We have just recovered Hawking's result for black hole lifetime!

Finally, let us consider using a black hole to do computations. This may sound like a ridiculous proposition. But if we believe that black holes evolve according to quantum mechanical laws, it is possible, at least in principle, to program black holes to perform computations that can be read out of the fluctuations in the Hawking black hole radiation. How large is the memory space of a black hole computer, and how fast can it compute? Applying the results for computation derived above, we readily find the number of bits in the memory space of a black hole computer, given by the lifetime of the black hole divided by its resolution time as a clock, to be

$$
I=\frac{T_{B H}}{t_{B H}} \sim \frac{m^{2}}{m_{P}^{2}} \sim \frac{r_{S}^{2}}{l_{P}^{2}}
$$

where $m_{P}=\hbar /\left(t_{P} c^{2}\right)$ is the Planck mass, $m$ and $r_{S}^{2}$ denote the mass and event horizon area of the black hole respectively. This gives the number of bits $I$ as the event horizon area in Planck units, in agreement with the identification of a black hole entropy. Furthermore, the number of operations per unit time for a black hole computer is given by

$$
I \nu \sim m c^{2} / \hbar,
$$

its energy divided by Planck's constant, in agreement with the result found by Margolus and Levitin, and by Lloyd 16 (for the ultimate limits to computation). It is curious that all the bounds on computation discussed above are saturated by black hole computers. Thus one can even say that once they are programmed to do computations, black holes are the ultimate simple computers.

All these results reinforce the conceptual interconnections of the physics underlying spacetime foam, black holes, and computation. It is intersting that these three 


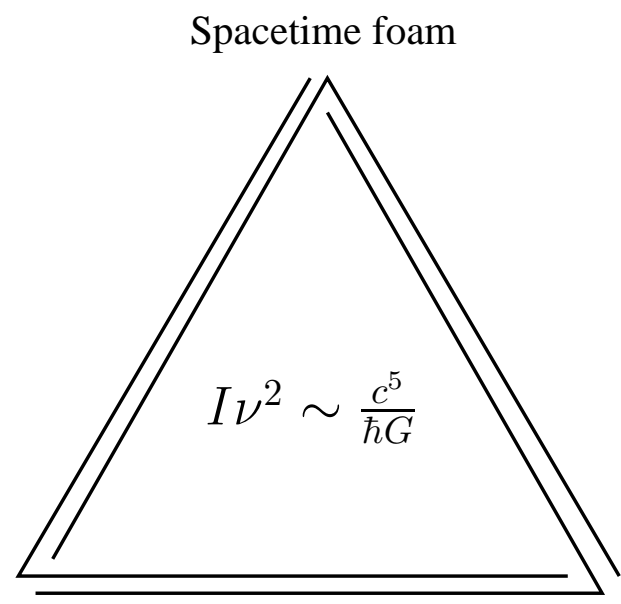

\section{Black hole}

\section{Computation/Information}

Figure 3. The quantum foam-black hole-computation/information triangle. At the center of the triangle is the universal relation: $I \nu^{2} \sim c^{5} / \hbar G$, where $I$ is the number of bits in the memory space, and $\nu$ is the clock rate of computation of a black hole computer. This relation is a combined product of the physics behind spacetime foam, black holes, and computation/information.

subjects share such intimate bonds and are brought together here [see Fig. 3]. The internal consistency of the physics we have uncovered also vindicates the simple (some would say overly simple) arguments we present in section 2 in the derivation of the limits to spacetime measurements.

\section{Energy-Momentum Uncertainties}

Just as there are uncertainties in spacetime measurements, there are also uncertainties in energy-momentum measurements due to spacetime foam effects. Thus there is a limit to how accurately we can measure and know the energy and momentum of a system. 1 Imagine sending a particle of momentum $p$ to probe a certain structure of spatial extent $l$ so that $p \sim \hbar / l$. It follows that $\delta p \sim\left(\hbar / l^{2}\right) \delta l$. Spacetime fluctuations $\delta l \gtrsim l\left(l_{P} / l\right)^{2 / 3}$ can now be used to give

$$
\delta p=\beta p\left(\frac{p}{m_{P} c}\right)^{2 / 3}
$$

where a priori $\beta \sim 1$. The corresponding statement for energy uncertainties is

$$
\delta E=\gamma E\left(\frac{E}{E_{P}}\right)^{2 / 3}
$$

where $E_{P}=m_{P} c^{2}$ is the Planck energy and a priori $\gamma \sim 1$. We emphasize that all the uncertainties take on \pm sign with equal probability (most likely, a Gaussian distribution about zero). Thus at energy-momentum far below the Planck scale, 
the energy-momentum uncertainties are very small, suppressed by a fractional (twothirds) power of the Planck energy-momentum. (For example, the uncertainty in the energy of a particle of ten trillion electron-volts is about a thousand electron-volts.)

Energy-momentum uncertainties affect both the energy-momentum conservation laws and the dispersion relations. Energy-momentum is conserved up to energymomentum uncertainties due to quantum foam effects, i.e., $\Sigma\left(p_{i}^{\mu}+\delta p_{i}^{\mu}\right)$ is conserved, with $p_{i}^{\mu}$ being the average values of the various energy-momenta. On the other hand the dispersion relation is now generalized to read

$$
E^{2}-p^{2} c^{2}-\epsilon p^{2} c^{2}\left(\frac{p c}{E_{P}}\right)^{2 / 3}=m^{2} c^{4}
$$

for high energies with $E \gg m c^{2}$. A priori we expect $\epsilon \sim 1$ and is independent of $\beta$ and $\gamma$. But due to our present ignorance of quantum gravity, we are not in a position to make any definite statements. In fact, it is possible that $\epsilon=2(\beta-\gamma)$, which would be the case if the modified dispersion relation is given by $(E+\delta E)^{2}-(p+\delta p)^{2} c^{2}=$ $m^{2} c^{4}$.

The modified dispersion relation discussed above has an interesting consequence for the speed of light!17.18 Applying Eq. (15) to the massless photon yields

$$
E^{2} \simeq c^{2} p^{2}+\epsilon E^{2}\left(\frac{E}{E_{P}}\right)^{2 / 3}
$$

The speed of (massless) photon

$$
v=\frac{\partial E}{\partial p} \simeq c\left(1+\frac{5}{6} \epsilon \frac{E^{2 / 3}}{E_{P}^{2 / 3}}\right),
$$

becomes energy-dependent and fluctuates around c. For example, a photon of ten trillion electron-volt energy has a speed fluctuating about $c$ by several centimeters per second.

\section{Spacetime Foam Phenomenology}

Because the Planck length $l_{P} \sim 10^{-33} \mathrm{~cm}$ is so minuscule, the Planck time $t_{P} \sim$ $10^{-44}$ sec so short, and the Planck energy $E_{P} \sim 10^{28} \mathrm{eV}$ so high, spacetime foam effects, suppressed by Planck scales, are exceedingly small. Accordingly, they are very hard to detect. The trick will be to find ways to amplify the small effects! 1

\subsection{Phase Incoherence of Light from Extra-galactic Sources}

One way to amplify the minute effects is to add up many such effects, like collecting many small raindrops to fill a reservoir. Consider light coming to us from extragalactic sources. Over one wavelength, the phase of the light-waves advances by $2 \pi$; but due to spacetime foam effects, this phase fluctuates by a small amount. The idea is that the fluctuation of the phase over one wavelength is extremely small, but 
light from distant galaxies has to travel a distance of many wavelengths. It is possible that over so many wavelengths, the fluctuations can cumulatively add up to a detectable level at which point the phase coherence for the light-waves is lost. Loss of phase coherence would mean the loss of interference patterns. Thus the strategy is to look for the blurring of images of distant galaxies in powerful telescopes like the Hubble Space Telescope. This technique to detect spacetime foam was proposed by Lieu and Hillman 19 , and elaborated by Ragazzoni and his collaborators 20 .

The proposal deals with the phase behavior of radiation with wavelength $\lambda$ received from a celestial source located at a distance $l$ away. Fundamentally, the wavelength defines the minimum length scale over which physical quantities such as phase and group velocities (and hence dispersion relations) can be defined. Thus, the uncertainty in $\lambda$ introduced by spacetime foam is the starting point for this analysis. A wave will travel a distance equal to its own wavelength $\lambda$ in a time $t=\lambda / v_{g}$ where $v_{g}$ is the group velocity of propagation, and the phase of the wave consequently changes by an amount

$$
\phi=2 \pi \frac{v_{p} t}{\lambda}=2 \pi \frac{v_{p}}{v_{g}}
$$

(i.e., if $v_{p}=v_{g}, \phi=2 \pi$ ) where $v_{p}$ is the phase velocity of the light wave. Quantum gravity fluctuations, however, introduce random uncertainties into this phase which is simply

$$
\delta \phi=2 \pi \delta\left(\frac{v_{p}}{v_{g}}\right)
$$

Due to quantum fluctuations of energy-momentum 4 and the modified dispersion relations, we obtain

$$
\delta\left(\frac{v_{p}}{v_{g}}\right) \sim \pm\left(\frac{E}{E_{P}}\right)^{2 / 3}= \pm\left(\frac{l_{P}}{\lambda}\right)^{2 / 3}
$$

where we have used $v_{p}=E / p$ and $v_{g}=d E / d p$, and $E / E_{P}=l_{P} / \lambda$. We emphasize that this may be either an incremental advance or a retardation in the phase.

In travelling over the macroscopically large distance, $l$, from source to observer an electromagnetic wave is continually subjected to random, incoherent spacetime fluctuations. Therefore, by our previous argument given in subsection 2.3, the cumulative statistical phase dispersion is $\Delta \phi=\mathcal{C} \delta \phi$ with the cumulative factor $\mathcal{C}=(l / \lambda)^{1 / 3}$, that is

$$
\Delta \phi=2 \pi a\left(\frac{l_{P}}{\lambda}\right)^{2 / 3}\left(\frac{l}{\lambda}\right)^{1 / 3}=2 \pi a \frac{l_{P}^{2 / 3} l^{1 / 3}}{\lambda},
$$

where $a \sim 1$. (This is our fundamental disagreement 13 with Lieu and Hillman who assume that the microscale fluctuations induced by quantum foam into the phase of electromagnetic waves are coherently magnified by the factor $l / \lambda$ rather than $(l / \lambda)^{1 / 3}$.) Thus even the active galaxy PKS1413+135, an example used by 
Lieu and Hillman, which is more than four billion light years from Earth, is not far enough to make the light wave front noticeably distorted. A simple calculation 13 shows that, over four billion light years, the phase of the light waves fluctuates only by one billionth of what is required to lose the sharp ring-like interference pattern around the galaxy which, not surprisingly, is observed 21 by the Hubble Telescope. This example illustrates the degree of difficulty which one has to overcome to detect spacetime foam. The origin of the difficulty can be traced to the incoherent nature of the spacetime fluctuations (i.e., the anticorrelations between successive fluctuations).

But not all is lost with Lieu and Hillman's proposal. One can check that the proposal can be used to rule out 13 , if only marginally, the random-walk model of quantum gravity, which would (incorrectly) predict a large enough phase fluctuation for light from PKS1413+135 to lose phase coherence, contradicting evidence of diffraction patterns from the Hubble Telescope observation. It follows that models corresponding to correlating successive fluctuations are also ruled out.

\subsection{High Energy $\gamma$ Rays from Distant GRB}

For another idea to detect spacetime foam, let us recall that, due to quantum fluctuations of spacetime, the speed of light fluctuates around $c$ and the fluctuations increase with energy. Thus for photons (quanta of light) emitted simultaneously from a distant source coming towards our detector, we expect an energy-dependent spread in their arrival times. To maximize the spread in arrival times, we should look for energetic photons from distant sources. High energy gamma rays from distant gamma ray bursts 17 fit the bill. So the idea is to look for a noticeable spread in arrival times for such high energy gamma rays from distant gamma ray bursts. This proposal was first made by G. Amelino-Camelia et al ${ }^{17}$ in another context.

To underscore the importance of using the correct cumulative factor to estimate the spacetime foam effect, let us first proceed in a naive manner. At first sight, the fluctuating speed of light would seem to yield $\frac{18}{18}$ an energy-dependent spread in the arrival times of photons of the same energy $E$ given by $\delta t \sim|\epsilon| t\left(E / E_{P}\right)^{2 / 3}$, where $t$ is the average overall time of travel from the photon source. Furthermore, the modified energy-momentum dispersion relation would seem to predict time-of-flight differences between simultaneously-emitted photons of different energies, $E_{1}$ and $E_{2}$, given by $\delta t \simeq \epsilon t\left(E_{1}^{2 / 3}-E_{2}^{2 / 3}\right) / E_{P}^{2 / 3}$. But these results for the spread of arrival times of photons are not correct, because we have inadvertently used $l / \lambda \sim E t / \hbar$ as the cumulative factor instead of the correct factor $(l / \lambda)^{1 / 3} \sim(E t / \hbar)^{1 / 3}$. Using the correct cumulative factor, we get a much smaller $\delta t \sim t^{1 / 3} t_{P}^{2 / 3}$ for the spread in arrival time of the photons of the same energy. Thus the result is that the timeof-flight differences increase only with the cube root of the average overall time of travel from the gamma ray bursts to our detector, leading to a time spread too small to be detectable ${ }^{1}$ 


\subsection{Interferometry Techniques}

Suppressed by the extraordinarily short Planck length, fluctuations in distances, even large distances, are very small. So, to measure such fluctuations, what one needs is an instrument capable of accurately measuring fluctuations in length over long distances. Modern gravitational-wave interferometers, having attained extraordinary sensitivity, come to mind. The idea of using gravitational-wave interferometers to measure the foaminess of spacetime was proposed by Amelino-Camelia 10 and elaborated by the author and van Dam 6 . Modern gravitational-wave interferometers are sensitive to changes in distances to an accuracy better than $10^{-18}$ meter. To attain such sensitivity, interferometer researchers have to contend with many different noises, the enemies of gravitational-wave research, such as thermal noise, seismic noise, and photon shot noise. To this list of noises that infest an interferometer, we now have to add the faint yet ubiquitous noise from spacetime foam. In other words, even after one has subtracted all the well-known noises, there is still the noise from spacetime fluctuations left in the read-out of the interferometer.

The secret of this proposal to detect spacetime foam lies in the existence of another length scale 10 available in this particular technique, in addition to the minuscule Planck length. It is the scale provided by the frequency $f$ of the interferometer bandwidth. What is important is whether the length scale $l_{P}^{2 / 3}(c / f)^{1 / 3}$, characteristic of the noise from spacetime foam at that frequency, is comparable to the sensitivity level of the interferometer. The hope is that, within a certain range of frequencies, the experimental limits will soon be comparable to the theoretical predictions for the noise from quantum foam.

The detection of spacetime foam with interferometry techniques is also helped by the fact that the correlation length of the noise from spacetime fluctuations is extremely short, as the characteristic scale is the Planck length. Thus, this faint noise can be easily distinguished from the other sources of noise because of this lack of correlation. In this regard, it will be very useful for the detection of spacetime foam to have two nearby interferometers.

To proceed with the analysis, one first decomposes the displacement noise in terms of the associated displacement amplitude spectral density $22 S(f)$ of frequency $f$. For the displacement noise due to quantum foam, it is given by $S(f) \sim c^{1 / 3} l_{P}^{2 / 3} f^{-5 / 6}$, inversely proportional to (the $5 / 6$ th power of) frequency. So one can optimize the performance of an interferometer at low frequencies. As lower frequency detection is possible only in space, interferometers like the proposed Laser Interferometer Space Antenna 23 may enjoy a certain advantage.

To be specific, let us now compare the predicted spectal density from quantum foam noise with the noise level projected for the Laser Interferometer GravitationalWave Observatory. The "advanced phase" of LIGO 24 is expected to achieve a displacement noise level of less than $10^{-20} \mathrm{mHz}^{-1 / 2}$ near $100 \mathrm{~Hz}$; one can show that this would translate into a probe of $l_{P}$ down to $10^{-31} \mathrm{~cm}$, a mere hundred times the physical Planck length. But can we then conclude that LIGO will be within striking 
distance of detecting quantum foam? Alas, the above optimistic estimate is based on the assumption that spacetime foam affects the paths of all the photons in the laser beam coherently. But, in reality, this can hardly be the case. Since the total effect on the interferometer is based on averaging over all photons in the wave front, the incoherent contributions from the different photons are expected to cut down the sensitivity of the interferometer by some fractional power of the number of photons in the beam - and there are many photons in the beams used by LIGO. Thus, even with the incredible sensitivity of modern gravitational-wave interferometers like LIGO, the fluctuations of spacetime are too small to be detected - unless one knows how to build a small beam interferometer of slightly improved power and phase sensitivity than what is projected for the advanced phase of LIGO! ${ }^{\text {e }}$

For completeness, we should mention that the use of atom interferometers 725 and optical interferometers 26 to look for effects of spacetime fluctuations has also been suggested.

Last but not least, spacetime foam physics has been applied to explain some baffling ultra-high energy cosmic ray events 27 reported by the Akeno Giant Air Shower Array observatory in Japan. But there are uncertainties on both the observational and theoretical sides. We relegate a short discussion on the UHECR events to the Appendix.

\section{Summary and Conclusion}

We summarize by collecting some of the salient points:

- On large scales spacetime appears smooth, but on a sufficiently small scale it is bubbly and foamy (just as the ocean appears smooth at high altitudes but shows its roughness at close distances from its surface).

- Spacetime is foamy because it undergoes quantum fluctuations which give rise to uncertainties in spacetime measurements; spacetime fluctuations scale as the cube root of distances or time durations.

- Quantum foam physics is closely related to black hole physics and computation. The "strange" holographic principle, which limits how densely information can be packed in space, is a manifestation of quantum foam.

- Because the Planck length/time is so small, the uncertainties in spacetime measurements, though much greater than the Planck scale, are still very small.

- It may be difficult to detect the tiny effects of quantum foam, but it is by no means impossible.

Recall that, by analyzing a simple gedanken experiment for spacetime measurements, we arrive at the conclusion that spacetime fluctuations scale as the cube root of distances or time durations. This cube root dependence is mysterious, but has

\footnotetext{
e This conclusion is based on the author's discussion with G. Amelino-Camelia and R. Weiss.
} 
been shown to be consistent with the holographic principle and with semi-classical black hole physics in general. Thus, to this author, this result for spacetime fluctuations is as beautiful as it is strange (and hopefully also true)! Perhaps Sir Francis Bacon was indeed right: There is no excellent beauty that hath not some strangeness in the proportion.

But strange beauty is no guarantee for experimental vindication. What is needed is direct detection of quantum foam. Its detection will give us a glimpse of the fabric of spacetime and will help guide physicists to the correct theory of quantum gravity. The importance of direct experimental evidence cannot be over-emphasized.

Now the ball is in the experimentalists' court.

\section{Acknowledgments}

This work was supported in part by the US Department of Energy and the Bahnson Fund of the University of North Carolina. Help from L. L. Ng in the preparation of this manuscript is gratefully acknowledged. I also thank my collaborators, especially H. van Dam and G. Amelino-Camelia, and R. Weiss for useful discussions. Thanks are due to B. Schumacher and M. Taqqu for conversations which led to the inclusion of the subsection on the various quantum gravity models.

\section{Appendix A. Ultra-high Energy Cosmic Ray Events}

The universe appears to be more transparent to the ultra-high energy cosmic rays (UHECRs) 27 than expected. ${ }^{\mathrm{f}}$ Theoretically one expects the UHECRs to interact with the Cosmic Microwave Background Radiation and produce pions. These interactions above the threshold energy should make observations of UHECRs with $E>5 \cdot 10^{19} \mathrm{eV}$ (the GZK limit) ${ }^{28}$ unlikely. Still UHECRs above the GZK limit have been observed. In this appendix, we attempt to explain the UHECR paradox by arguing 18 that energy-momentum uncertainties due to quantum gravity (significant only for high energy particles like the UHECRs), too small to be detected in low-energy regime, can affect particle kinematics so as to raise or even eliminate the energy thresholds, thereby explaining the threshold anomaly. ${ }^{\mathrm{g}}$ (For similar or related approaches, see Ref. 29.)

Relevant to the discussion of the UHECR events is the scattering process in which an energetic particle of energy $E_{1}$ and momentum $\mathbf{p}_{1}$ collides head-on with a soft photon of energy $\omega$ in the production of two energetic particles with energy $E_{2}, E_{3}$ and momentum $\mathbf{p}_{2}, \mathbf{p}_{3}$. After taking into account energy-momentum uncertainties, energy-momentum conservation demands

$$
E_{1}+\delta E_{1}+\omega=E_{2}+\delta E_{2}+E_{3}+\delta E_{3}
$$

${ }^{\mathrm{f}}$ For the case of (the not-so-well-established) $\mathrm{TeV}-\gamma$ events, see Ref. 1.

g Unfortunately, we have nothing useful to say about the origins of these energetic particles per se. 
and

$$
p_{1}+\delta p_{1}-\omega=p_{2}+\delta p_{2}+p_{3}+\delta p_{3},
$$

where $\delta E_{i}$ and $\delta p_{i}(i=1,2,3)$ are given by Eqs. (14) and (13),

$$
\delta E_{i}=\gamma_{i} E_{i}\left(\frac{E_{i}}{E_{P}}\right)^{2 / 3}, \delta p_{i}=\beta_{i} p_{i}\left(\frac{p_{i}}{m_{P} c}\right)^{2 / 3},
$$

and we have omitted $\delta \omega$, the contribution from the uncertainty of $\omega$, because $\omega$ is small. ${ }^{\mathrm{h}}$

Combining Eq. A.3 with the modified dispersion relations ${ }^{\mathrm{i}}$ Eq. (15) for the incoming energetic particle $(i=1)$ and the two outgoing particles $(i=2,3)$, and putting $c=1$,

$$
E_{i}^{2}-p_{i}^{2}-\epsilon_{i} p_{i}^{2}\left(\frac{p_{i}}{E_{P}}\right)^{2 / 3}=m_{i}^{2}
$$

we obtain the threshold energy equation

$$
E_{t h}=p_{0}+\tilde{\eta} \frac{1}{4 \omega} \frac{E_{t h}^{8 / 3}}{E_{P}^{2 / 3}},
$$

where

$$
p_{0} \equiv \frac{\left(m_{2}+m_{3}\right)^{2}-m_{1}^{2}}{4 \omega}
$$

is the (ordinary) threshold energy if there were no energy-momentum uncertainties, and

$$
\tilde{\eta} \equiv \eta_{1}-\frac{\eta_{2} m_{2}^{5 / 3}+\eta_{3} m_{3}^{5 / 3}}{\left(m_{2}+m_{3}\right)^{5 / 3}}
$$

with

$$
\eta_{i} \equiv 2 \beta_{i}-2 \gamma_{i}-\epsilon_{i}
$$

Note that, in Eq. A.5 , the quantum gravity correction term is enhanced by the fact that $\omega$ is so small 131 (compared to $p_{0}$ ).

Given that all the $\beta_{i}$ 's, the $\gamma_{i}$ 's and the $\epsilon_{i}$ 's are of order 1 and can be,$\pm \tilde{\eta}$ can be \pm (taking on some unknown Gaussian distribution about zero), but it cannot be much bigger than 1 in magnitude. For positive $\tilde{\eta}, E_{t h}$ is greater than $p_{0}$. The threshold energy increases with $\tilde{\eta}$ to $\frac{3}{2} p_{0}$ at $\tilde{\eta}=\tilde{\eta}_{\text {max }}$, beyond which there is no (real) physical solution to Eq. (A.5.e., $E_{t h}$ becomes complex) and we interpret this as evading the threshold cut $\frac{18}{18}$ The cutoff $\tilde{\eta}_{\max }$ is actually very small: $\tilde{\eta}_{\max } \sim 10^{-17}$.

${ }^{\mathrm{h}}$ We should mention that we have not found the proper (possibly nonlinear) transformations of the energy-momentum uncertainties between different reference frames. Therefore we apply the results only in the frame in which we do the observations.

iThe suggestion that the dispersion relation may be modified by quantum gravity first appeared in Ref. 30. 
Thus, energy-momentum uncertainties due to quantum gravity, too small to be detected in low-energy regime, can (in principle) affect particle kinematics so as to raise or even eliminate energy thresholds. Can this be the solution to the UHECR threshold anomaly puzzle? On the other hand, for negative $\tilde{\eta}$, the threshold energy is less than $p_{0}$, i.e., a negative $\tilde{\eta}$ lowers the threshold energy $[23233$ Can this be the explanation of the opening up of the "precocious" threshold in the "knee" region? Curiously, the interpolation between the "knee" region and the GKZ limit may even explain the "ankle" region. 1]

It is far too early to call this a success. In fact there are some problems confronting this particular proposal to solve the astrophysical puzzle. The most serious problem is the question of matter (in)stability 34 because quantum fluctuations in dispersion relations Eq. (A.4) can lower as well as raise the reaction thresholds. This problem may force us to entertain one or a combination of the following possibilities: (i) The fluctuations of the energy-momentum of a particle are not completely uncorrelated (e.g, the fluctuating coefficients $\beta, \gamma$, and $\epsilon$ in Eqs. (13), (14), and (15) may be related such that $\eta_{i} \approx 0$ in Eq. (A.8)); (ii) The time scale at which quantum fluctuations of energy-momentum occur is relatively short ${ }^{\mathrm{j}}$ (compared to the relevant interaction or decay times); (iii) Both "systematic" and "non-systematic" effects

of quantum gravity are present, 2 but the "systematic" effects are large enough to overwhelm the "non-systematic" effects.

\section{References}

1. Y. J. Ng, Mod. Phys. Lett. A18, 1073 (2003).

2. G. Amelino-Camelia, Y.J. Ng, H. van Dam, gr-qc/0204077, Astropart. Phys. 19, 729 (2003).

3. E.P. Wigner, Rev. Mod. Phys. 29, 255 (1957); H. Salecker and E.P. Wigner, Phys. Rev. 109, 571 (1958).

4. Y.J. Ng and H. van Dam, Mod. Phys. Lett. A9, 335 (1994); A10, 2801 (1995); in Proc. of Fundamental Problems in Quantum Theory, eds. D.M. Greenberger and A. Zeilinger, Ann. New York Acad. Sci. 755, 579 (1995). Also see F. Karolyhazy, Nuovo Cimento A42, 390 (1966); D.V. Ahluwalia, Phys. Lett. B339, 301 (1994); and N. Sasakura, Prog. Theor. Phys. 102, 169 (1999).

5. L. H. Ford, Phys. Rev. D51, 1692 (1995).

6. Y. J. Ng and H. van Dam, Found. Phys. 30, 795 (2000); Phys. Lett. B477, 429 (2000).

7. Y. J. Ng, Int. J. Mod. Phys. D11, 1585 (2002).

8. G. 't Hooft, in Salamfestschrift, edited by A. Ali et al. (World Scientific, Singapore, 1993), p. 284; L. Susskind, J. Math. Phys. (N.Y.) 36, 6377 (1995). Also see J.A. Wheeler, Int. J. Theor. Phys. 21, 557 (1982); J.D. Bekenstein, Phys. Rev. D7, 2333 (1973); S. Hawking, Comm. Math. Phys. 43, 199 (1975).

9. F. Scardigli and R. Casadio, hep-th/0307174, Class. Quant. Grav. 20, 3915 (2003).

$\overline{\mathrm{j} U n f o r t u n a t e l y, ~ t h e s e ~ t w o ~ s c e n a r i o s ~ a l s o ~ p r e c l u d e ~ t h e ~ p o s s i b i l i t y ~ t h a t ~ e n e r g y-m o m e n t u m ~ u n c e r t a i n-~}$ ties are the origin of the threshold anomaly discussed above. On the positive side, the threshold anomaly suggested by the present AGASA data may turn out to be false. Data from the Auger Project are expected to settle the issue. 
10. G. Amelino-Camelia, Nature 398, 216 (1999).

11. L. Diosi and B. Lukacs, Phys. Lett. A142, 331 (1989).

12. C. W. Misner, K. S. Thorne, and J. A. Wheeler, Gravitation (Freeman, San Francisco, 1973), 1190

13. Y. J. Ng, W. Christiansen, and H. van Dam, astro-ph/0302372 Astrophys. J. 591, L87 (2003).

14. Y. J. Ng, Phys. Rev. Lett. 86, 2946 (2001), and (erratum) 88, 139902-1 (2002); Y. J. Ng in Proc. of OCPA 2000, eds. N. P. Chang et al. (World Scientific, Singapore, 2002), p.235.

15. J.D. Barrow, Phys. Rev. D54, 6563 (1996).

16. N. Margolus and L. B. Levitin, Physica D120, 188 (1998). S. Lloyd, Nature (London) 406, 1047 (2000).

17. G. Amelino-Camelia, J. Ellis, N.E. Mavromatos, D.V. Nanopoulos, and S. Sarkar, Nature 393, 763 (1998); B. E. Schaefer, Phys. Rev. Lett. 82, 4964 (1999); S.D. Biller et al., ibid, 83, 2108 (1999).

18. Y. J. Ng, D. S. Lee, M. C. Oh, and H. van Dam, Phys. Lett. B507, 236 (2001); hep-ph/0010152 and references therein. The arXiv preprint is a more informative version of the paper in Phys. Lett. B.

19. R. Lieu and L. W. Hillman, astro-ph/0301184 Astrophys. J. 585, L77 (2003).

20. R. Ragazzoni, M. Turatto, and W. Gaessler, astro-ph/0303043 Astrophys. J. 587, L1 (2003).

21. E. S. Perlman, et al., 2002, Astro. J. 124, 2401 (2002).

22. V. Radeka, Ann. Rev. Nucl. Part. Sci. 38, 217 (1988).

23. K. Danzmann, Class. Quant. Grav. 13, A247 (1996).

24. A. Abramovici, et. al., Science 256, 325 (1992).

25. I. Percival, Phys. World, March 1997, p.43; F. Benatti and R. Floreanini, quant-ph/0208164

26. F. Benatti and R. Floreanini, quant-ph/0204094 L.B. Crowell, Found. Phys. 16, 281 (2003).

27. M.A. Lawrence et al., J. Phys. G17, 733 (1991); N. N. Efimov et al., in 22nd Intl. Cosmic Ray Conf. (Dublin, 1991); D.J. Bird et al., Astrophys. J. 441, 144 (1995); M. Takeda et al., Phys. Rev. Lett. 81, 1163 (1998); A. Watson, in Proc. Snowmass Workshop, 126 (1996).

28. K. Greisen, Phys. Rev. Lett. 16, 748 (1966); G. T. Zatsepin and V.A. Kuz'min, JETP Lett. 41, 78 (1966).

29. C.J. Cesarsky, Nucl. Phys. (Proc. Suppl.) B28, 51 (1992); L. Gonzalez-Mestres, physics/9704017 R. Aloisio, P. Blasi, P.L. Ghia, and A.F. Grillo, astro-ph/0001258 O. Bertolami and C.S. Carvalho, Phys. Rev. D61, 103002 (2000); H. Sato, astro-ph/0005218 T. Kifune, Astrophys. J. Lett. 518, L21 (1999); W. Kluzniak, astro-ph/9905308 S. Coleman and S.L. Glashow, Phys. Rev. D59, 116008 (1999); D. Colladay and A. Kostelecky, Phys. Rev. D55, 6760 (1997); R. Lieu, ApJ 568, L67 (2002). Also see F.W. Stecker, astro-ph/0304527

30. G. Amelino-Camelia, J. Ellis, N.E. Mavromatos, and D.V. Nanopoulos, Int. J. Mod. Phys. A12, 607 (1997).

31. G. Amelino-Camelia abd T. Piran, hep-th/0006210 astro-ph/0008107

32. R. Aloisio, P. Blasi, A. Galante, P.L. Ghia, and A.F. Grillo, Astropart. Phys. 19, 127 (2003).

33. Y.J. Ng, talk given in the Huntsville Workshop 2002 (unpublished).

34. R. Aloisio, P. Blasi, A. Galante, and A.F. Grillo, astro-ph/0304050 R. Le Gallou, astro-ph/0304560 